This is the author's copy of the publication as archived with the DLR's electronic library at http://elib.dlr.de . Please consult the original publication for citation.

\title{
Model Predictive Power Allocation for Hybrid Battery Balancing Systems
}

\author{
R. de Castro and R. E. Araujo
}

This work focuses on hybrid balancing systems, a recently-proposed concept that enables balancing of battery cells and hybridization with supercapacitors. To control this system, a model predictive control framework is developed. In addition to distributing the supercapacitor power among the balancing circuits, this framework is also able to minimize state-of-charge and thermal imbalances in the battery cells, as well as energy losses in the balancing circuits. The effectiveness of the proposed approach is verified via numerical simulations. It is shown that, in comparison with state-of-art balancing solutions, the proposed control approach is able to decrease battery stress in up to $9 \%$ and the maximum temperature in up to $4.5 \%$.

\section{Copyright Notice}

(C2020 IEEE. Personal use of this material is permitted. Permission from IEEE must be obtained for all other uses, in any current or future media, including reprinting/republishing this material for advertising or promotional purposes, creating new collective works, for resale or redistribution to servers or lists, or reuse of any copyrighted component of this work in other works.

R. de Castro and R. E. Araujo, "Model Predictive Power Allocation for Hybrid Battery Balancing Systems," 2019 IEEE Vehicle Power and Propulsion Conference (VPPC), Hanoi, Vietnam, 2019, pp. 1-5, doi: 10.1109/VPPC46532.2019.8952557. 


\title{
Model Predictive Power Allocation for Hybrid Battery Balancing Systems
}

\author{
Ricardo de Castro*, Rui Esteves Araujo ${ }^{\dagger}$, \\ * Institute of System Dynamics and Control, German Aerospace Center (DLR), Wessling, D-82234, Germany \\ $\dagger$ INESC TEC and Faculty of Engineering, University of Porto, Porto, 4200-465, Portugal \\ e-mail: Ricardo.DeCastro@dlr.de
}

\begin{abstract}
This work focuses on hybrid balancing systems, a recently-proposed concept that enables balancing of battery cells and hybridization with supercapacitors. To control this system, a model predictive control framework is developed. In addition to distributing the supercapacitor power among the balancing circuits, this framework is also able to minimize state-of-charge and thermal imbalances in the battery cells, as well as energy losses in the balancing circuits. The effectiveness of the proposed approach is verified via numerical simulations. It is shown that, in comparison with state-of-art balancing solutions, the proposed control approach is able to decrease battery stress in up to $9 \%$ and the maximum temperature in up to $4.5 \%$.
\end{abstract}

\section{INTRODUCTION}

Batteries are one of the key elements for the successful deployment of electric vehicles (EVs). As discussed in several technological roadmaps [1], [2], EVs are pushing the performance goals of batteries, demanding higher durability, fast charging capability, reduced volume and mass, and lower cost. However, to achieve these goals, several challenges need to be addressed. One of the main challenges is the parameter variations present in battery packs, which appear due to unavoidable manufacturing tolerances and non-uniform aging of battery cells. These variations, together with large series connections of battery cells (typical in battery packs of EVs), introduce the so-called weakest-cell problem, i.e., cells with weaker thermal and capacity properties limit the performance of the entire battery pack [3]. Battery balancing systems, capable of equalizing voltage, state-of-charge and/or temperature of battery cells, are a promising solution to mitigate the weakestcell problem [4], [5].

Another challenge in the design of battery packs lies in the selection of battery chemistries capable of simultaneously offering high energy density, high power density and long life. This difficulty has motivated the development of hybrid energy storage systems that combine batteries and supercapacitors [6]. The idea is to integrate energy storage units with complementary characteristics - batteries with high energy density and supercapacitors with high power density- such that the resulting hybrid energy storage system provides better overall performance [7], [8].

This work deals with hybrid balancing systems (HBSs), where battery balancing and hybridization functions share the same power converters. Fig. 1 shows one possible implementation of a HBS -reliant on a cell-to-cell shared configuration [9]- that allows the transfer of energy between: $i$ ) different battery modules and $i i)$ supercapacitors and battery modules. Thanks to this integrated power conversion, energy management and power allocation \& battery balancing can be simultaneously performed. The energy management determines the power split between batteries and supercapacitors that minimize energy losses and battery stress. The power allocation \& battery balancing allocates the supercapacitor power to the HBS balancing circuits and equalizes state-ofcharge and temperature of the battery modules.

The main contribution of this work consists in the exploitation of control-allocation methods to tackle the power allocation \& battery balancing problem of HBSs. In particular, we propose a model-predictive power allocation (MPPA) method to distribute the supercapacitor power between the balancing circuits of the HBS, while minimizing state-ofcharge and thermal imbalances in the battery modules. This approach is particularly attractive, because of the MPPA's capability to handle multi-variable control problems, like the HBS, and fulfillment of physical actuation constraints. To the best of the author's knowledge, this represents one of the first contributions toward the decoupled control of HBSs.

\section{Problem Formulation}

\section{A. Overview of the $H B S$}

Fig. 1 depicts the hybrid energy storage system, composed of batteries and supercapacitors, under consideration in this work. The battery pack consists of a series of $n$ modules, each one containing one or more cells in parallel. The battery modules are connected with $n$ bidirectional balancing circuits (based on dual half bridge converters) that allow the transfer of energy between supercapacitors and battery modules.

The control architecture of the balancing circuits is divided into three layers. The first layer, energy management, determines the amount of power $\left(p_{S C}^{*}\right)$ that the supercapacitors should provide to the batteries taking into account energy efficiency and battery stress metrics. The second layer, power allocation \& battery balancing, computes setpoints for the balancing currents $i_{B, j}^{*}, j=1, \ldots, n$, such that $i$ ) the supercapacitor power requested by the energy management $\left(p_{S C}^{*}\right)$ is executed, and $\left.i i\right)$ thermal and state-of-charge (SoC) variations in the battery modules are minimized. The third layer consists of inner current loops ${ }^{1}$ that control the balancing

\footnotetext{
${ }^{1}$ in Fig. 1, the inner current loops are integrated into the balancing circuits and are not explicitly depicted
} 


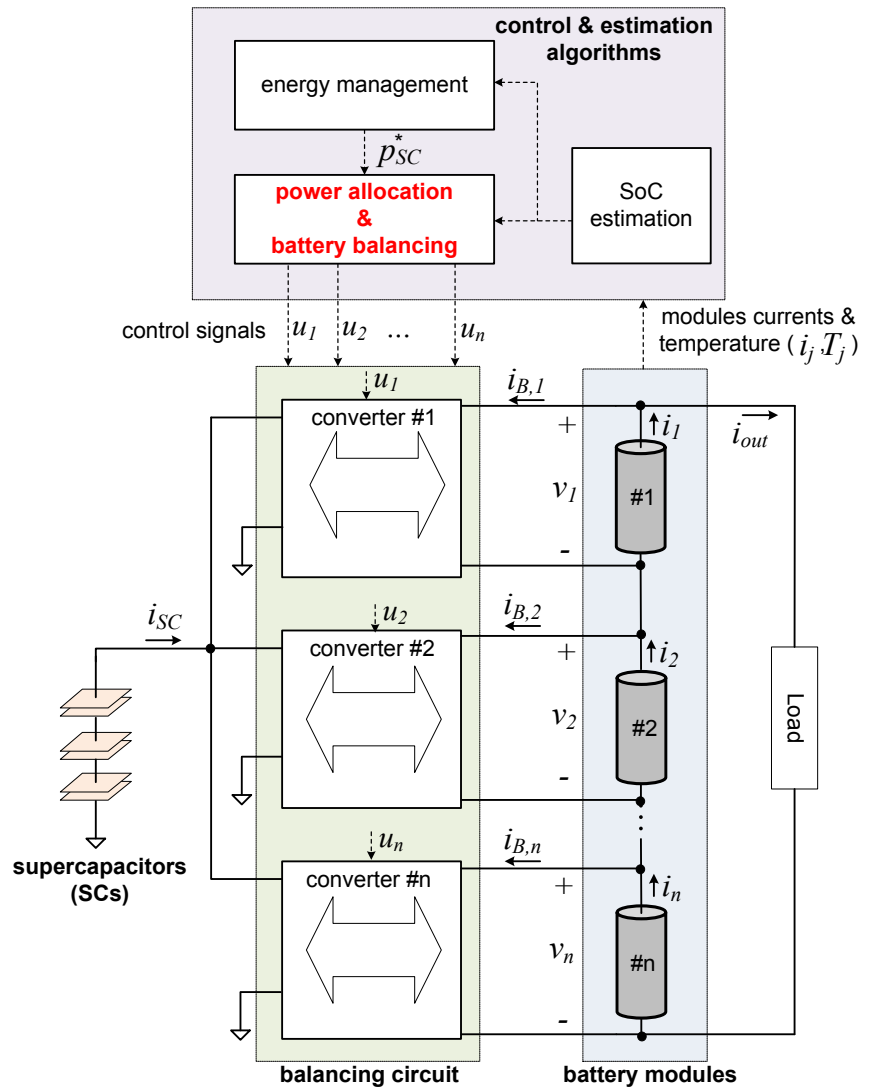

Fig. 1. Block diagram of the hybrid energy storage system ( batteries and supercapacitors), and hybrid balancing system ( balancing circuit and control). The control signals sent to the balancing circuit represent setpoints for the balancing currents $\left(u_{j}=i_{B, j}^{*}\right)$.

currents $i_{B, j}$ of the HBS. In the sequel, we will focus on the design of the power allocation \& battery balancing layer. The supercapacitor reference $p_{S C}^{*}$ is assumed to be available, e.g. using stat-of-the-art energy management methods [6]. Similarly, it is also assumed that balancing circuits are endowed with inner-current loops (see [10] for details).

\section{B. Modeling}

This section presents control-oriented models for the HBS. Accordingly, the electro-thermal model of the battery modules is described as [9]

$$
\begin{aligned}
\dot{q}_{j} & =-\frac{1}{\bar{Q}_{j}} i_{j} \\
v_{j} & =f_{j}\left(q_{j}\right)-R_{j} i_{j} \\
C_{h, j} \dot{T}_{j} & =R_{j} i_{j}^{2}-\frac{1}{R_{c o v, j}}\left(T_{j}-T_{e n v}\right)
\end{aligned}
$$

for $j=1, \ldots, n$. The first equation represents the $\operatorname{SOC}\left(q_{j}\right)$ dynamics, where $\bar{Q}_{j}$ is the nominal capacity and $i_{j}$ the current in the battery module $j$. The second equation approximates the terminal voltage $\left(v_{j}\right)$ of the battery module $j$ using the opencircuit voltage source $f_{j}\left(q_{j}\right)$-dependent on the SoC-in series with an internal resistance $\left(R_{j}\right)$. The third equation captures the thermal response of the battery module, where $T_{j}$ is the surface temperature, $T_{e n v}$ the environmental temperature, $C_{h, j}$ the thermal capacitance and $R_{c o v, j}$ the convective thermal resistance.

As shown in Fig. 1, the battery modules are connected in parallel with the vehicle load, i.e. the vehicle's powertrain. The power requested by this load is related with the battery voltage and load current $\left(i_{\text {out }}\right)$ through the relation

$$
p_{\text {out }}=\sum_{j} v_{j} i_{\text {out }}
$$

while the load current is further linked with the battery and balancing currents through Kirchhoff's current law

$$
i_{j}=i_{B, j}+i_{\text {out }}
$$

The balancing circuits are modeled here as ideal dc-dc transformers. This means that the power extracted by the supercapacitors $\left(p_{S C}\right)$ should be transferred to the battery modules and cover the power losses of the converters, which yields:

$$
p_{S C}=-\sum_{j} v_{j} i_{B, j}+R_{B, j} i_{B, j}^{2}
$$

where $R_{B, j}$ captures the energy losses in the balancing circuit.

This work neglects the transient response of the inner current loops, i.e. $i_{B, j}^{*} \approx i_{B, j}$. This is justified by the large time-scale separation that exists between the fast response of the inner current loops (order of microseconds) and the slower response of the battery SoC and temperature (order of seconds and minutes).

To obtain a compact representation of the HBS, we stack the SoC, temperatures, and voltages of the battery modules into the vectors $\mathbf{q}, \mathbf{v}, \mathbf{T}$, and treat the balancing currents $\left(i_{B, j}\right)$ as control variables $(\mathbf{u})$ :

$$
\begin{aligned}
\mathbf{q} & =\left[q_{1} \ldots q_{n}\right]^{T}, \quad \mathbf{v}=\left[v_{1} \ldots v_{n}\right]^{T}, \\
\mathbf{T} & =\left[T_{1} \ldots T_{n}\right]^{T}, \quad \mathbf{u}=\left[i_{B, 1} \ldots i_{B, n}\right]^{T}
\end{aligned}
$$

This leads to the following nonlinear state-space model

$$
\begin{aligned}
\dot{\mathbf{q}} & =\mathbf{B}_{q}\left(\mathbf{u}+\mathbf{1} i_{\text {out }}\right) \\
\mathbf{v} & =\mathbf{f}(\mathbf{q})-\mathbf{B}_{v}\left(\mathbf{u}+\mathbf{1} i_{\text {out }}\right) \\
\dot{\mathbf{T}} & =\mathbf{A}_{T}\left(\mathbf{T}-\mathbf{1} T_{\text {env }}\right)+\mathbf{B}_{T} \mathbf{g}\left(\mathbf{u}+\mathbf{1} i_{\text {out }}\right) \\
p_{\text {out }} & =\mathbf{v}^{T} \mathbf{1} i_{\text {out }} \\
p_{S C} & =-\mathbf{v}^{T} \mathbf{u}+\mathbf{r}_{B}^{T} \mathbf{g}(\mathbf{u})
\end{aligned}
$$

where $\mathbf{g}$ is a quadratic mapping, $\mathbf{1} \in \mathbb{R}^{n}$ a column vector filled with ones, $\mathbf{f}$ a vector of open-circuit voltages and $\mathbf{B}_{q}, \mathbf{A}_{T}, \mathbf{B}_{T}, \mathbf{B}_{v}, \mathbf{r}_{B}$ matrices dependent on the battery and balancing circuit parameters. Table I presents the definition of all matrices and functions employed in this state-state model.

\section{Control Requirements and Goals}

The balancing currents are constrained by physical limits in the power converters:

$$
\mathcal{U}=\left\{\mathbf{u} \in \mathbb{R}^{n}: \mathbf{u}_{\min } \leq \mathbf{u} \leq \mathbf{u}_{\max }\right\}
$$

where $\mathbf{u}_{\min }, \mathbf{u}_{\max }$ represents the minimum and maximum balancing currents allowed by power converters in the HBS. 
TABLE I

MATRICES OF THE HBS MODEL

\begin{tabular}{cl}
\hline Variable & Value \\
\hline $\mathbf{B}_{q}$ & $\operatorname{diag}\left(-\frac{1}{\bar{Q}_{1}}, \ldots,-\frac{1}{\bar{Q}_{n}}\right)$ \\
$\mathbf{A}_{T}$ & $\operatorname{diag}\left(-\frac{1}{C_{h, 1} R_{\operatorname{cov}, 1}}, \ldots,-\frac{1}{C_{h, n} R_{\text {cov }, n}}\right)$ \\
$\mathbf{B}_{T}$ & $\operatorname{diag}\left(-\frac{R_{1}}{C_{h, 1}}, \ldots,-\frac{R_{n}}{C_{h, n}}\right)$ \\
$\mathbf{B}_{v}$ & $\operatorname{diag}\left(R_{1}, \ldots, R_{n}\right)$ \\
$\mathbf{r}_{B}$ & {$\left[R_{b, 1} \ldots R_{b, n}\right]^{T}$} \\
$\mathbf{f}(\mathbf{q})$ & {$\left[f_{1}\left(q_{1}\right) \ldots f_{n}\left(q_{n}\right)\right]^{T}$} \\
$\mathbf{g}(\mathbf{u})$ & {$\left[u_{1}^{2} \ldots u_{n}^{2}\right]^{T}$} \\
$\mathbf{1}$ & {$[1 \ldots 1]^{T}$} \\
\hline
\end{tabular}

To promote equalization of the battery modules, it is desirable to operate with small SoC variations [5]. These variations are usually defined as differences with respect to the average behavior of the battery pack, i.e., $\Delta q_{i}=q_{i}-\tilde{q}$, where $\tilde{q}=\frac{1}{n} \mathbf{1}^{T} \mathbf{q}$ is the average SoC in the battery pack. This is equivalent to:

$$
\Delta \mathbf{q}=\left(\mathbf{I}-\frac{1}{n}\left[\begin{array}{c}
\mathbf{1}^{T} \\
\vdots \\
\mathbf{1}^{T}
\end{array}\right]\right) \mathbf{q}=\mathbf{M} \mathbf{q}
$$

where $\Delta \mathbf{q}=\left[\Delta q_{1}, \ldots, \Delta q_{n}\right]^{T}$. Similarly, it is also helpful to minimize thermal imbalances in the battery pack. These imbalances are defined as $\Delta T_{i}=T_{i}-\tilde{T}$, where $\tilde{T}=\frac{1}{n} \mathbf{1}^{T} \mathbf{T}$ is the average temperature in the pack; this is equivalent to

$$
\Delta \mathbf{T}=\mathbf{M T}
$$

where $\Delta \mathbf{T}=\left[\Delta T_{1}, \ldots, \Delta T_{n}\right]^{T}$.

The main problem of this work can now be formulated as:

Problem 1. Consider the nonlinear state-space model (9) for the HBS. Find the balancing currents $\mathbf{u} \in \mathcal{U}$ that

(a) deliver the supercapacitor power requested by energy management layer:

$$
p_{S C}^{*}=-\mathbf{v}^{T} \mathbf{u}+\mathbf{r}_{B}^{T} \mathbf{g}(\mathbf{u})
$$

(b) minimize SoC imbalances: $\|\Delta \mathbf{q}\| \rightarrow 0$

(c) minimize thermal imbalances: $\|\Delta \mathbf{T}\| \rightarrow 0$

(d) minimize energy losses in the balancing circuits: $\mathbf{u}^{T} \mathbf{R}_{B} \mathbf{u} \rightarrow 0$

where $\mathbf{R}_{B}=\operatorname{diag}\left(\mathbf{r}_{B}\right)$.

\section{Power Allocation AND BATTERy BALANCING}

This section describes a model predictive approach to solve the power allocation \& battery balancing problem introduced in the previous section.

\section{A. Motivation}

According to Problem 1, the allocation of the supercapacitor power is considered as the primary control goal and encoded as a hard constraint (13). Since this constraint is composed of one nonlinear equation with $n$ unknown variables (i.e., the vector $\mathbf{u}$ ), multiple solutions might exist [11]. For example,
TABLE II

PARAMETERS OF THE HBS

\begin{tabular}{cccc}
\hline Variable & Symbol & Value & Unit \\
\hline $\begin{array}{c}\text { Battery pack } \\
\text { initial SoC }\end{array}$ & $\mathbf{q}_{0}$ & {$[0.9,0.92,0.88]$} & $\mathrm{V}$ \\
nominal capacity & $\bar{Q}_{j}$ & {$[4,3,3] \times 3$} & $\mathrm{~A} . \mathrm{h}$ \\
internal resistance & $R_{\text {bat }}$ & {$\left[\frac{1}{4}, \frac{1}{3}, \frac{1}{3}\right] \times 51.16$} & $\mathrm{~m} \Omega$ \\
open-circuit voltage & $f_{j}$ & $3.207+0.33 q_{j}$ & $\mathrm{~V}$ \\
stored energy & & $10 \times 10.8$ & $\mathrm{~W} . \mathrm{h}$ \\
\hline $\begin{array}{c}\text { Supercapacitors } \\
\text { stored energy }\end{array}$ & & & \\
Thermal Parameters & & $4 \times 0.31$ & $\mathrm{~W} . \mathrm{h}$ \\
thermal capacitance & $C_{h}$ & {$[161,125,125]$} & $\mathrm{J} / \mathrm{K}$ \\
convective resistance & $R_{\text {cov }, j}$ & {$[10.3,13.7,13.7]$} & $\mathrm{K} / \mathrm{W}$ \\
environment temperature & $T_{\text {env }}$ & 25 & ${ }^{\circ} \mathrm{C}$ \\
\hline $\begin{array}{c}\text { Balancing circuit } \\
\text { inner resistance }\end{array}$ & $R_{B, j}$ & {$[0.04,0.04,0.04]$} & $\Omega$ \\
\hline Balancing parameters & & & \\
balancing current limits & $\mathbf{u}_{\max }$ & {$[5,5,5]$} & $\mathrm{A}$ \\
& $\mathbf{u}_{\min }$ & $-[5,5,5]$ & $\mathrm{A}$ \\
controller weights & $\Delta q_{\max }$ & 0.01 & {$[-]$} \\
& $\Delta T_{\max }$ & 0.75 & ${ }^{\circ} \mathrm{C}$ \\
& $\alpha_{u}$ & 0.5 & {$[-]$} \\
prediction horizon & $N$ & 10 & {$[-]$} \\
sample time & $\tau_{s}$ & 1 & $\mathrm{~s}$ \\
\hline
\end{tabular}

one can allocate the supercapacitor power to a single balancing circuit $^{2}$ or evenly split it among the balancing converters ${ }^{3}$. This multitude of solutions can be combined with optimizationbased strategies to pursue secondary control goals, such as minimization of energy losses, SoC and thermal imbalances, as described in Problems 1b, 1c and 1d. In the literature, this type of problem is known as control allocation problem and is usually formulated as a constrained optimization problem. Since our secondary control goals depend on dynamic variables such as SoC $\mathbf{q}$ and temperature $\mathbf{T}$ - we adopt here a modelpredictive framework, inspired by [12], to solve Problem 1. We call this approach model-predictive power allocation (MPPA).

\section{B. $M P P A$}

In order to design the MPPA, let us first discretize the model (9) using Euler's method and sample time $\tau_{s}$

$$
\begin{aligned}
\mathbf{q}[k+1] & =\mathbf{q}[k]+\tau_{s} \mathbf{B}_{q}\left(\mathbf{u}[k]+\mathbf{1} i_{\text {out }}[k]\right) \\
\mathbf{v}[k] & =\mathbf{f}(\mathbf{q}[k])-\mathbf{B}_{v}\left(\mathbf{u}[k]+\mathbf{1} i_{\text {out }}[k]\right) \\
\mathbf{T}[k+1]= & \mathbf{T}[k]+\tau_{s} \mathbf{A}_{T}\left(\mathbf{T}[k]-\mathbf{1} T_{\text {env }}[k]\right) \\
& +\tau_{s} \mathbf{B}_{T} \mathbf{g}\left(\mathbf{u}[k]+\mathbf{1} i_{\text {out }}[k]\right) \\
p_{\text {out }}[k]= & \mathbf{v}^{T}[k] \mathbf{1} i_{\text {out }}[k] \\
p_{S C}^{*}[k]= & -\mathbf{v}^{T}[k] \mathbf{u}[k]+\mathbf{r}_{B}^{T} \mathbf{g}(\mathbf{u}[k])
\end{aligned}
$$

where $k$ is the discrete time index.

The MPPA uses the above discrete model to predict the response of the HBS over a $N$-step horizon. It then maps the

$$
\begin{aligned}
& { }^{2} p_{S C}^{*}=-v_{l}^{T} u_{l}+R_{B, l} u_{l}^{2} \text { and } u_{j}=0 \text { for } j \neq l \\
& { }^{3} p_{S C}^{*} / n=-v_{j}^{T} u_{j}+R_{B, j} u_{j}^{2} \text { for } j=1, \ldots, n
\end{aligned}
$$


primary and secondary control goals, defined in Problem 1, into the following optimization problem:

$$
\begin{aligned}
\min _{\mathbf{u}[.]} \sum_{k=1}^{N} \Delta \mathbf{q}[k]^{T} \mathbf{Q}_{q} \Delta \mathbf{q}[k]+\Delta \mathbf{T}[k]^{T} \mathbf{Q}_{T} \Delta \mathbf{T}[k] & +\mathbf{u}[k]^{T} \mathbf{R u}[k] \\
\text { s.t. } & (14), \quad \mathbf{u}[k] \in \mathcal{U}, \\
& \Delta \mathbf{q}[k]=\mathbf{M q}[k], \quad \Delta \mathbf{T}[k]=\mathbf{M T}[k] \\
& \mathbf{q}[0]=\mathbf{q}_{0}, \quad \mathbf{T}[0]=\mathbf{T}_{0}, \quad k=0, \ldots, N
\end{aligned}
$$

The first set of constraints deals with the discrete model and physical actuation limits, the second computes the SoC and thermal variations, and the third enforces initial conditions. Regarding the cost function: the first two terms penalize SoC and thermal imbalances over the prediction horizon, while the last term penalizes energy losses in the balancing circuit; the matrices $\left(\mathbf{Q}_{q}, \mathbf{Q}_{T}, \mathbf{R}\right)$ are tuning weights, selected by the designer. To facilitate the tuning of these weights, the following normalization factors are introduced

$$
\mathbf{Q}_{q}=\Delta q_{\max }^{-2} \mathbf{I}, \quad \mathbf{Q}_{T}=\Delta T_{\max }^{-2} \mathbf{I}, \quad \mathbf{R}=\alpha_{u} \mathbf{R}_{B}
$$

where $\Delta q_{\max }$ represents the desired maximum SoC variation, $\Delta T_{\max }$ the desired maximum temperature variation and $\mathbf{I}$ the identity matrix. The parameter $\alpha_{u} \geq 0$ is a factor that allows the designer to explore trade-offs between energy losses and SoC/thermal imbalances. As a result of these normalization factors, the MPPA performance is ultimately dictated by the tuple $\left(\Delta q_{\max }, \Delta T_{\max }, \alpha_{u}\right)$.

We assume that the MPPA has access to the system states, either via measurement $(\mathbf{T})$ or estimation (q) [13]. The variables $p_{S C}^{*}[k], p_{\text {out }}[k], T_{\text {env }}[k]$ are treated here as known exogenous inputs during the prediction horizon, which allows the incorporation of preview information in the optimization problem. The MPPA implementation follows a receding horizon strategy, i.e. at each time step the optimal solution of (15) is computed $\left(\mathbf{u}^{*}[k]\right)$ and then the first element $\left(\mathbf{u}^{*}[0]\right)$ applied to the HBS. Because of nonlinear constraints, e.g. (14e), finding unique optimal solutions for the MPPA is a challenge task. To assist in the search of optimal solutions, we employed the IPOPT [14], an interior-point nonlinear programming solver.

\section{VALIDATION RESUlTS}

\section{A. Simulation Setup}

This section presents validation results of the MPPA-based HBS using numerical simulations. The model of the HBS is based on a small scale prototype composed of 10 battery cells (cylindrical Li-ion LG 18650HG2), 4 SCs (Maxwell BCAP0310 P270 T10) and 3 balancing circuits. The battery cells were organized in a string of 3 modules: module \#1 has 4 cells in parallel, while module \#2 and \#3 have 3 cells in parallel. This arrangement, which was also considered in our previous work [9], emulates a situation where the battery pack

\begin{tabular}{|c|c|c|c|c|}
\hline \multicolumn{5}{|c|}{ 1: average rms value of SoC differences } \\
\hline \multicolumn{5}{|c|}{$\Delta q_{r m s}=\frac{1}{n}\left(\Delta q_{1, r m s}+\ldots \Delta q_{n, r m s}\right)$} \\
\hline \multicolumn{5}{|c|}{ 2: average rms value of temperature differences } \\
\hline \multicolumn{5}{|c|}{$\Delta T_{r m s}=\frac{1}{n}\left(\Delta T_{1, r m s}+\ldots \Delta T_{n, r m s}\right)$} \\
\hline \multicolumn{5}{|c|}{ 3: maximum temperature in the battery modules } \\
\hline \multicolumn{5}{|c|}{$T_{\max }=\max _{j, k} T_{j}[k]$} \\
\hline \multicolumn{5}{|c|}{ 4: minimum SoC at the end of the driving cycles } \\
\hline \\
\hline & & & & $\begin{array}{l}\text { 5: average rms value of the battery current, } \\
\qquad i_{r m s}=\underline{1}\left(i_{1}\right.\end{array}$ \\
\hline \\
\hline \multicolumn{5}{|c|}{$i_{j, r m s}=\sqrt{\frac{1}{M} \sum_{k=1}^{M} i_{j}^{2}[k] \tau_{s}}$} \\
\hline \multirow{2}{*}{\multicolumn{5}{|c|}{$\begin{array}{l}M=\text { duration of the driving cycle } \\
\text { rms = root-mean-square }\end{array}$}} \\
\hline & & & & \\
\hline \multirow{2}{*}{\multicolumn{5}{|c|}{$\begin{array}{c}\text { TABLE IV } \\
\text { VALUE OF PERFORMANCE METRICS }\end{array}$}} \\
\hline & & & & \\
\hline & No & Active & Hybrid & Hybrid \\
\hline & $\begin{array}{l}\text { Balancing } \\
\text { (NB) }\end{array}$ & $\begin{array}{c}\text { Balancing } \\
(\mathrm{AB})\end{array}$ & $\begin{array}{l}\text { No Balancing } \\
\text { (HNB) }\end{array}$ & $\begin{array}{l}\text { Balancing } \\
\text { (HBS) }\end{array}$ \\
\hline \multicolumn{5}{|l|}{ equalization } \\
\hline $100 \times \Delta q_{r m s}$ & $4.03 \%$ & $0.45 \%$ & $3.93 \%$ & $0.48 \%$ \\
\hline$\Delta T_{r m s}$ & $1.66^{\circ} \mathrm{C}$ & $0.44^{\circ} \mathrm{C}$ & $1.36^{\circ} \mathrm{C}$ & $0.37^{\circ} \mathrm{C}$ \\
\hline \multicolumn{5}{|l|}{ thermal/SOC } \\
\hline$T_{\max }$ & $38.10^{\circ} \mathrm{C}$ & $36.10^{\circ} \mathrm{C}$ & $35.80^{\circ} \mathrm{C}$ & $34.20^{\circ} \mathrm{C}$ \\
\hline $100 \times q_{\min }$ & $27.66 \%$ & $35.62 \%$ & $29.74 \%$ & $37.34 \%$ \\
\hline \multicolumn{5}{|l|}{ battery stress } \\
\hline$i_{r m s}$ & $7.72 \mathrm{~A}$ & $7.75 \mathrm{~A}$ & $6.97 \mathrm{~A}$ & $6.99 \mathrm{~A}$ \\
\hline$i_{1, r m s}$ & $7.72 \mathrm{~A}$ & $8.87 \mathrm{~A}$ & $6.97 \mathrm{~A}$ & $8.00 \mathrm{~A}$ \\
\hline$i_{2, \mathrm{rms}}$ & $7.72 \mathrm{~A}$ & $7.23 \mathrm{~A}$ & $6.97 \mathrm{~A}$ & $6.54 \mathrm{~A}$ \\
\hline$i_{3, r m s}$ & $7.72 \mathrm{~A}$ & $7.13 \mathrm{~A}$ & $6.97 \mathrm{~A}$ & $6.42 \mathrm{~A}$ \\
\hline
\end{tabular}
has significant imbalances in capacity and inner resistance. The parameter values of the battery modules are present in Table II. To transfer energy between the battery modules and SCs,
TABLE III

DEFINITION OF PERFORMANCE METRICS

Note: best values are highlighted in blue.

balancing circuits based on dual half bridge converters were considered. These converters employs phase-shift methods to control the balancing currents (see [10] for details).

The power requested to the energy storage system follows a scaled version of the US06 driving cycle, repeated 6 times and with a power scaling factor ${ }^{4}$ of 1 to 140 . This driving cycle information is explored by the energy management layer to compute the supercapacitor power setpoint $\left(p_{S C}^{*}\right)$ that minimizes energy losses in the hybrid energy storage system, using an optimization-based methodology similar to [9]. Besides the MPPA-based HBS, three additional variants were simulated:

- no balancing $(\mathrm{NB})$ with $\mathbf{u}=0$

- active balancing (AB), obtained by solving the MPPA problem (15) without the supercapacitor presence $\left(p_{S C}^{*}=\right.$ $0)$.

- hybridization and no balancing (HNB), obtained by solving the MPPA problem (15) parameterized with $\Delta q_{\max }=\infty, \Delta T_{\max }=\infty$

\section{B. Performance Metrics}

To quantify benefits of the HBS, several key performance metrics related with the equalization goals, thermal behavior, SoC discharge and battery stress were computed -see Table III

\footnotetext{
${ }^{4}$ reference vehicle for power calculation is the uCar [8]
} 
for their definition. Inspecting the value of these metrics, represented in Table IV, reveals that the HBS provides: $i$ ) similar equalization performance as the $\mathrm{AB} ; i i$ ) similar average battery stress as the HNB and; iii) the best thermal behavior and SoC discharge metrics. In other words, the HBS combines the strengths of the active balancing and hybridization to produce the best overall performance. More specifically, the HBS, thanks to the power aid provided by the supercapacitors, is able to reduce the average root-mean-square current in the battery to 6.99Arms (a 9.4\% decrease in comparison with $\mathrm{NB}$ and $\mathrm{AB}$ ), which might lessen battery degradation. This stress reduction also decreases the maximum temperature of the battery pack to $34.2^{\circ} \mathrm{C}$ (i.e., $-3.9^{\circ} \mathrm{C}$ than the $\mathrm{NB}$ configuration, $-1.9^{\circ} \mathrm{C}$ than the $\mathrm{AB}$ and $-1.6^{\circ} \mathrm{C}$ than $\mathrm{HNB}$ ), which decreases the cooling needs of the battery. Finally, the HBS exhibits the highest SoC at the end of the driving cycle (9.6\% higher than the NB, $1.7 \%$ higher than the $\mathrm{AB}$ and $7.6 \%$ higher than HNB), which contributes to an increase in the vehicle's range.

\section{CONCLUSIONS}

This paper introduced a new control method for hybrid balancing systems. It exploited a decoupled control architecture, which divided the energy management and power allocation \& battery balancing into two separate control layers. A systematic framework was then developed to model the hybrid balancing system and solve the power allocation \& battery balancing with model-predictive and control allocation methods. Simulation results demonstrated that, in comparison with other state-of-art balancing systems, the proposed concept was able to simultaneously improve equalization, thermal, SoC and battery stress performance metrics. In future works, we plan to experimentally validate the proposed control method and evaluate its robustness against parameter uncertainty.

\section{ACKNOWLEDGMENT}

The work of Rui. E. Araújo was funded by FCT, Fundação para a Ciência e a Tecnologia, project UID/EEA/50014/2019.

\section{REFERENCES}

[1] ERTRAC, "European Roadmap Electrification of Road Transport," Tech. Rep., 2017.

[2] H. Hendrikse, V. Greer, J. Yeung, A. Jonas, B. Singh, Y. Shin, and H. Isozaki, "Electric Vehicles: On the Charge," Morgan Stanley, Tech. Rep., 2017.

[3] J. Barreras, D. Frost, and D. Howey, "Smart Balancing Systems: An Ultimate Solution to the Weakest Cell Problem?" IEEE Vehicular Technology Society Newsletter, 2018.

[4] D. F. Frost and D. A. Howey, "Completely Decentralized Active Balancing Battery Management System," IEEE Transactions on Power Electronics, vol. 33, no. 1, pp. 729-738, Jan. 2018.

[5] F. Altaf, L. Johannesson, and B. Egardt, "Simultaneous Thermal and State-of-Charge Balancing of Batteries: A Review," in IEEE Vehicle Power and Propulsion Conference (VPPC), Oct. 2014.

[6] Ahmed Ali and Dirk Söffker, "Towards Optimal Power Management of Hybrid Electric Vehicles in Real-Time: A Review on Methods, Challenges, and State-Of-The-Art Solutions," Energies, vol. 11, no. 3, p. 476 , Feb. 2018

[7] K. Alobeidli and V. Khadkikar, "A New Ultracapacitor State of Charge Control Concept to Enhance Battery Lifespan of Dual Storage Electric Vehicles," IEEE Transactions on Vehicular Technology, vol. 67, no. 11, pp. 10470-10481, Nov. 2018.
[8] R. E. Araujo, R. de Castro, C. Pinto, P. Melo, and D. Freitas, "Combined Sizing and Energy Management in EVs With Batteries and Supercapacitors," IEEE Transactions on Vehicular Technology, vol. 63, no. 7, pp. 3062-3076, Sep. 2014.

[9] C. Pinto, R. de Castro, J. V. Barreras, R. E. Araujo, and D. Howey, "Smart Balancing Control of a Hybrid Energy Storage System based on a Cell-to-Cell Shared Energy Transfer Configuration," in IEEE Vehicle Power and Propulsion Conference, Chicago, USA, 2018.

[10] C. Pinto, "Sizing and Energy Management of a Distributed Hybrid Energy Storage System for Electric Vehicles," PhD. Dissertation, University of Porto, 2018.

[11] T. A. Johansen and T. I. Fossen, "Control allocation: a survey," Automatica, vol. 49, no. 5, pp. 1087-1103, 2013

[12] M. Hanger, T. A. Johansen, G. K. a. Mykland, and A. Skullestad, "Dynamic model predictive control allocation using CVXGEN," in 9th IEEE International Conference on Control and Automation (ICCA), 2011, pp. 417-422.

[13] W. Waag, C. Fleischer, and D. U. Sauer, "Critical review of the methods for monitoring of lithium-ion batteries in electric and hybrid vehicles," Journal of Power Sources, vol. 258, pp. 321-339, Jul. 2014.

[14] A. Wachter and L. T. Biegler, "On the implementation of an interiorpoint filter line-search algorithm for large-scale nonlinear programming," Mathematical Programming, vol. 106, no. 1, pp. 25-57, Mar. 2006. 